\title{
EDITORIAL \\ 6 Toward reducing futile recanalization in stroke: automated prediction of final infarct volume
}

\author{
Muhammad Waqas, MBBS, ${ }^{1,2}$ and Elad I. Levy, MD, MBA ${ }^{1-5}$ \\ Departments of ${ }^{1}$ Neurosurgery and ${ }^{3}$ Radiology, Jacobs School of Medicine and Biomedical Sciences, and ${ }^{4}$ Canon Stroke and \\ Vascular Research Center, University at Buffalo; ${ }^{2}$ Department of Neurosurgery, Gates Vascular Institute at Kaleida Health, \\ Buffalo; and ${ }^{5}$ acobs Institute, Buffalo, New York
}

$\mathrm{T}$ HE premise of endovascular revascularization is to rescue the brain tissue at risk of ischemic infarct in the presence of large-vessel occlusion. Given a considerable rate of futile revascularization (defined as unfavorable clinical outcomes despite successful reperfusion), efforts have been made to improve the selection of patients eligible for mechanical thrombectomy. ${ }^{1}$ Final infarct volume (FIV) is probably the most significant determinant of unfavorable clinical outcomes in patients undergoing mechanical thrombectomy. Advancements in neuroimaging and image postprocessing have provided neurointerventionalists with tools to optimize patient selection based on the volume of the ischemic penumbra and completed infarct. Eligibility criteria for thrombectomy patients have evolved over the years to include perfusion imaging. An extension of the eligibility time window for treatment has been a direct result of CT perfusion (CTP) imaging and automated estimation of the penumbra and infarct. ${ }^{2,3}$ This has been perhaps the most noticeable change in the eligibility criteria for mechanical thrombectomy after the publication of several successful clinical trials. ${ }^{4}$

Most research on preprocedureal assessment of the penumbra and infarct is based on CTP imaging. Software such as Rapid (iSchemaView) and Viz.ai (Viz.ai, Inc.) has become part of the routine workflow at several centers across the US. CT angiography (CTA) and noncontrast head CT (NCHCT) have been shown to predict FIV; however, few studies have described automated processing of the CTA to predict FIV.

In this article, Abdelkhaleq and coauthors evaluated 3 measures of infarct core estimation at presentation: NCHCT, Alberta Stroke Program Early CT Score (ASPECTS), CTA (DeepSymNet [DSN]), and CTP (Rapid). ${ }^{5}$ The authors have done commendable work by introducing a novel machine learning algorithm for automated processing and prediction of FIV using CTA.
This is a multicenter collaboration of four centers that included 76 patients in whom CT-ASPECTS, CTA-DSN, and CTP-Rapid had been concurrently performed, and who had presented within 6 hours of symptom onset. The median time between when the patient was last known to be well and arrival was 156 minutes (range 73-303 minutes), and from NCHCT, CTA, and CTP to groin puncture was 73 minutes (range 54-81 minutes). Receiver operating characteristic (ROC) curve analysis was performed to estimate the predictive value of ASPECTS, CTA, and CTP-Rapid. The area under the ROC curve (AUC) was obtained at three different cutoff points: $10-\mathrm{ml}, 30-\mathrm{ml}$, and 50-ml FIV. The authors found that at the 10-ml FIV cutoff, the AUC of the ASPECTS was 0.67; of the CTP core volume, 0.63; and of DSN, $0.75(\mathrm{p}=0.11)$. At the $30-\mathrm{ml}$ FIV cutoff, the AUC of the ASPECTS was 0.76; of the CTP core volume, 0.73; and of DSN, $0.82(p=0.36)$. At the 50-ml FIV cutoff, the AUC of the ASPECTS was 0.74; of the CTP core volume, 0.72; and of the DSN, 0.82. Although DSN showed better AUCs for all three thresholds, these differences in AUCs were not significant $(p=0.44)$.

Key aspects of the paper are the use of a binary outcome for ROC analysis (i.e., FIV of less than or more than $50 \mathrm{ml}, 30 \mathrm{ml}$, and $10 \mathrm{ml}$ ). These thresholds were chosen based on reports in the literature and distribution of the data. The study is difficult to compare with other studies performed to assess the predictive value of CTP for FIV because most CTP studies do not use a binary outcome for FIV. Instead, they correlate core volume on CTP imaging with postthrombectomy infarct volume on fluid-attenuated inversion recovery or diffusion-weighted images of the brain. It will be interesting to study the correlation of infarct volume derived from preprocedural CTA-DSN with FIV seen on postthrombectomy MRI and compare it with the correlation of CTP.

Although the sample size of the study is sufficient for 
proof of concept, larger studies are needed with external validation and with patients presenting more than 6 hours after symptom onset. Because perfusion-based selection of patients for mechanical thrombectomy is particularly relevant to patients presenting after 6 hours, any modality that predicts FIV needs to be validated in this subgroup of patients. Another important future consideration is the ability of CTA to estimate the volume of the penumbra. An estimation of the mismatch ratio will clearly increase the practical value of such an algorithm.

As the field of neurointervention continues to embrace modern technology, artificial intelligence and machine learning will likely have a greater role in the diagnosis, management, and prognostication of patients with largevessel occlusion acute ischemic stroke.

https://thejns.org/doi/abs/10.3171/2021.4.FOCUS21263

\section{References}

1. Meinel TR, Kaesmacher J, Mosimann PJ, et al. Association of initial imaging modality and futile recanalization after thrombectomy. Neurology. 2020;95(17):e2331-e2342.

2. Nogueira RG, Jadhav AP, Haussen DC, et al. Thrombectomy 6 to 24 hours after stroke with a mismatch between deficit and infarct. N Engl J Med. 2018;378(1):11-21.
3. Albers GW, Marks MP, Kemp S, et al. Thrombectomy for stroke at 6 to 16 hours with selection by perfusion imaging. N Engl J Med. 2018;378(8):708-718.

4. Goyal M, Menon BK, van Zwam WH, et al. Endovascular thrombectomy after large-vessel ischaemic stroke: a metaanalysis of individual patient data from five randomised trials. Lancet. 2016;387(10029):1723-1731.

5. Abdelkhaleq R, Kim Y, Khose S, et al. Automated prediction of final infarct volume in patients with large-vessel occlusion acute ischemic stroke. Neurosurg Focus. 2021;51(1):E13.

\section{Disclosures}

Dr. Levy: ownership in NeXtGen Biologics, RAPID Medical, Claret Medical, Cognition Medical, Imperative Care, Rebound Therapeutics, StimMed, and Three Rivers Medical; and honoraria from Claret Medical, GLG, Guidepoint Global, Imperative Care, Medtronic, Rebound, StimMed, Misionix, Mosiac, Clarion, and IRRAS.

\section{Correspondence}

Elad I. Levy: elevy@ubns.com.

INCLUDE WHEN CITING

DOI: 10.3171/2021.4.FOCUS21263. 